\title{
One-Parameter Families of Optimization Problems: Equality Constraints
}

\author{
H. TH. JONGEN, ${ }^{1,2}$ P. JONKER, ${ }^{1}$ AND F. TWILT ${ }^{1}$ \\ Communicated by A. V. Fiacco
}

\begin{abstract}
In this paper, we introduce generalized critical points and discuss their relationship with other concepts of critical points [resp., stationary points]. Generalized critical points play an important role in parametric optimization. Under generic regularity conditions, we study the set of generalized critical points, in particular, the change of the Morse index. We focus our attention on problems with equality constraints only and provide an indication of how the present theory can be extended to problems with inequality constraints as well.
\end{abstract}

Key Words. Parametric optimization, generalized critical points, critical points, Morse index, quadratic index, linear index.

\section{Introduction}

Let $C^{k}\left(\mathbb{R}^{n}, \mathbb{R}\right), k \geq 1$ or $k=\infty$, denote the space of real-valued, $k$-times continuously differentiable functions defined on the $n$-dimensional Euclidean space $\mathbb{R}^{n}, n \geq 1$. Given finite index sets $I, J$, a differentiable optimization problem $P$ has the following standard formulation:

$(P)$ : minimize $f$, subject to $M[h, g]$,

where $f, h_{i}, g_{j} \in C^{2}\left(\mathbb{R}^{n}, \mathbb{R}\right), i \in I, j \in J$, and

$$
M[h, g]=\left\{x \in \mathbb{R}^{n} \mid h_{i}(x)=0, g_{j}(x) \geq 0, i \in I, j \in J\right\} .
$$

The function $f$ is the objective function, $h_{i}$ are the equality constraints, $g_{j}$ are the inequality constraints, and the set $M[h, g]$ is the feasible region. The activity map $J_{0}: \mathbb{R}^{n} \rightarrow \mathscr{P}(J), \mathscr{P}(J)$ being the power set of $J$, is defined by

$$
x \mapsto\left\{j \in J \mid g_{j}(x)=0\right\}
$$

\footnotetext{
'Senior Lecturer, Department of Applied Mathematics, Twente University of Technology, Enschede, The Netherlands.

${ }^{2}$ Professor, University of Hamburg, Hamburg, Germany.
} 
Thus, the set $J_{0}(x)$ corresponds to the set of those inequality constraints which are active at $x$. For $\phi \in C^{2}\left(\mathbb{R}^{n}, \mathbb{R}\right)$, we denote by $D \phi(x)$ [resp., $\left.D^{2} \phi(x)\right]$ the row vector of the first partial derivatives [resp., the symmetric $n \times n$-matrix of the second partial derivatives of $f]$, evaluated at $x$.

Definition 1.1. A point $\bar{x} \in \mathbb{R}^{n}$ is called a generalized critical point (g.c. point) for $P$ or $f_{\mid M[k, g]}$, if $\bar{x} \in M[h, g]$ and if the set $\left\{D f, D h_{i}, D g_{j}, i \in\right.$ $\left.I, j \in J_{0}(\bar{x})\right\}_{\mid x=\bar{x}}$ is linearly dependent.

The concept of a g.c. point encompasses, in a certain sense, every notion of stationary, critical, etc., points for $P$. Moreover, it turns out to be the right concept when dealing with parametric optimization problems.

Let $\bar{x}$ be a g.c. point for $P$. Then, there exist reals $\lambda, \lambda_{i}, \mu_{j}, i \in I, j \in J_{0}(\bar{x})$, not all vanishing, such that

$$
\lambda D f=\sum_{i \in I} \lambda_{i} D h_{i}+\sum_{j \in J_{0}(\bar{x})} \mu_{j} D g_{j \mid x=\bar{x}} .
$$

Of course, $\left(\lambda, \lambda_{i}, \mu_{j}\right)$ in (3) are unique--up to a common multiple-if and only if $\operatorname{dim} \operatorname{span}\left\{D f, D h_{i}, D g_{j}, i \in I, j \in J_{0}(\bar{x})\right\}_{\mid x=\bar{x}}$ equals $|I|+\left|J_{0}(\bar{x})\right|$, where $|\cdot|$ stands for the cardinality. If $\left(\lambda, \lambda_{i}, \mu_{j}\right)$ in (3) can be chosen such that $\lambda>0, \mu_{j} \geq 0, j \in J_{0}(\bar{x})\left[\right.$ resp., $\lambda \geq 0, \mu_{j} \geq 0, j \in J_{0}(\bar{x})$ ], then $\bar{x}$ is usually called a Kuhn-Tucker point [resp., a point of Fritz John type]. In both cases, local optimality criteria up to second order are well known (Refs. 1 and 2). In particular, a local minimum for $P$ must be a point of Fritz John type. However, a local minimum $\bar{x}$ for $P$ need not to be a Kuhn-Tucker point, unless some kind of a constraint qualification (of first order) at $\bar{x}$ is assumed. The simplest constraint qualification is to require the linear independence of the set $\left\{D h_{i}, D g_{j}, i \in I, j \in J_{0}(\bar{x})\right\}_{\mid x=\bar{x}}$. In the latter case it is easily shown that $M[h, g]$, locally around $\bar{x}$, is $C^{2}$-diffeomorphic with a neighborhood of the origin in $\mathbb{H}^{p} \times \mathbb{R}^{q}$, where

$$
p=\left|J_{0}(\bar{x})\right|, \quad q=n-|I|-p,
$$

and

$$
\mathbb{H}^{p}=\left\{\left(x_{1}, \ldots, x_{p}\right) \in \mathbb{R}^{p} \mid x_{i} \geq 0, i=1, \ldots, p\right\} .
$$

This means that we may replace the set $M[h, g]$, locally around $\bar{x}$, by the "linear set" $\mathbb{M}^{p} \times \mathbb{R}^{q}$, by means of a $C^{2}$-coordinate transformation.

Definition 1.2. The set $M:=M[h, g]$ is called regular at $\bar{x}, \bar{x} \in M$, if $\left\{D h_{i}, D g_{j}, i \in I, j \in J_{0}(\bar{x})\right\}_{\mid x=\bar{x}}$ is a linearly independent set. A generalized critical point $\bar{x}$ is called a critical point (for $P$ or for $f_{\mid M}$ ) if $M$ is regular at $\bar{x}$. 
If $\bar{x}$ is a critical point for $P$, then $\lambda, \lambda_{i}, \mu_{j}$ in (3) are unique if we put $\lambda=1$. In case $J=\varnothing$, the concept of a critical point coincides with the concept of a critical point for differentiable functions on differentiable manifolds (see Ref. 3). Let $A$ be a real symmetric $n \times n$ matrix, $L$ a linear subspace of $\mathbb{R}^{n}$, and $V$ a matrix whose columns ( $\mathbb{R}^{n}$ vectors) form a basis for $L$. As a consequence of Sylvester's theorem (Ref. 4), the number of negative [resp., zero, positive] eigenvalues of $V^{\top} A V$ is independent of the choice of the matrix $V$. By $A_{\mid L}$ we mean $V^{\top} A V$ for some matrix $V$ as above.

Definition 1.3. Let $\bar{x}$ be a critical point for $P$. Then, we may write (3) as follows:

$$
D f=\sum_{i \in I} \lambda_{i} D h_{i}+\sum_{j \in J_{0}(\bar{x})} \mu_{j} D g_{j \mid x=\bar{x}} .
$$

The critical point $\bar{x}$ is said to be nondegenerate, if conditions ND1, ND2 below hold:

(ND1): $\mu_{j} \neq 0, j \in J_{0}(\bar{x})$;

(ND2): $\quad D^{2} L(\bar{x})_{\mid T}$ is nonsingular,

where $L(x)$ is the Lagrange function

$$
L(x)=f(x)-\sum_{i \in I} \lambda_{i} h_{i}(x)-\sum_{j \in J_{0}(\bar{x})} \mu_{j} g_{j}(x)
$$

and

$$
T=\left\{\xi \in \mathbb{R}^{n} \mid D h_{i} \xi=D g_{j} \xi=0, i \in I, j \in J_{0}(\bar{x})\right\}_{\mid x=\bar{x}} .
$$

Definition 1.4. Let $\bar{x}$ be a nondegenerate critical point for $P$. The linear index (coindex) LI(LCI) at $\bar{x}$ is defined to be the number of those $\mu_{j}$ in (5) which are negative (positive). The quadratic index (coindex) QI(QCI) at $\bar{x}$ denotes the number of negative (positive) eigenvalues of $D^{2} L(\bar{x})_{\mid T}$ in (6).

Obviously, a nondegenerate critical point $\bar{x}$ for $P$ is a local minimum (maximum) for $f_{\mid M[h, g]}$ if and only if $\mathrm{LI}+\mathrm{QI}=0(\mathrm{LCI}+\mathrm{QCI}=0)$. If $J=\varnothing$, the concept of quadratic index coincides with the Morse index (Ref. 3). If $L I=0$, the quadratic index is equal to the $s$-index in the terminology of Kojima (Ref. 5). Moreover, if $\mathrm{LI}=0$ [resp., $\mathrm{LCI}=0$ ], the nondegenerate critical point is a $(+)$ critical point [resp., $(-)$ critical point] in the sense of Braess (Ref. 6). Note that nondegenerate critical points are isolated.

Definition 1.5. The set $M:=M[h, g]$ is called regular if it is regular at every point $x \in M$. The problem $P$ (or $f_{\mid M}$ ) is said to be regular if $M$ is regular and all critical points for $f_{\mid M}$ are nondegenerate. 
For a detailed study on global aspects of regular optimization problems, such as the study of lower level sets, relations between critical points, etc., we refer to Ref. 7.

We endow $C^{k}\left(\mathbb{R}^{n}, \mathbb{R}\right)$, for an $r \leq k$, with the strong (or Whitney) $C_{S}^{r}$-topology (Ref. 8), the $C_{S}^{r}$-topology for a finite product of copies of $C^{k}\left(\mathbb{R}^{n}, \mathbb{R}\right)$ being the induced product topology.

Theorem 1.1. Let $I, J$ be fixed finite index sets, and let $n \geq 1$. Put

$$
\mathscr{F}=\left\{\left(f, h_{i}, i \in I, g_{j}, j \in J\right) \in C^{2}\left(\mathbb{R}^{n}, \mathbb{R}\right)^{1+|I|+|J|} \mid f_{\mid M[h, g]} \text { is regular }\right\} .
$$

Then, $\mathscr{F}$ is $C_{S^{-}}^{2}$ open and dense in $C^{2}\left(\mathbb{R}^{n}, \mathbb{R}\right)^{1+|I|+|J|}$.

The proof of the open part of Theorem 1.1 is straightforward. The dense part within the class of $C^{\infty}$-functions is basically proved by means of Sard's theorem (Ref. 8). Since $C^{\infty}\left(\mathbb{R}^{n}, \mathbb{R}\right)$ is $C_{S}^{r}$-dense in $C^{k}\left(\mathbb{R}^{n}, \mathbb{R}\right)$ for all $r \leq k$ (Ref. 8), the dense part in Theorem 1.1 follows. We emphasize that the statement of Theorem 1.1 contains both an approximation aspect and a stability aspect. For a detailed discussion on the latter subject, we refer to Ref. 9. Note that $M[h, g]$ is always empty if $\left(f, h_{i}, i \in I, g_{j}, j \in J\right) \in \mathscr{F}$ and $|I|>n$.

After the above preliminaries, we pass on to optimization problems which depend on a real parameter, say $t$. Let $f, h_{i}, g_{j}, i \in I, j \in J$, be $C^{2}$-functions of $n+1$ variables, the last variable being denoted by $t$. Every $t$ defines an optimization problem $P(t)$ :

$$
\begin{aligned}
{[P(t)]: } & \text { minimize } f(\cdot, t), \text { subject to } M(t), \\
& \text { where } M(t)=M[h(\cdot, t), g(\cdot, t)] .
\end{aligned}
$$

For a special fixed value of $t$, say $\bar{t}$, it is not unreasonable to assume, in view of Theorem 1.1, that $P(\bar{t})$ is regular. However, as $t$ varies in $\mathbb{R}$, for certain values of $t$ the problem $P(t)$ need not to be regular. In fact, the following types of degeneracy may occur:

(a) $M(t)$ fails to be regular at some point $\bar{x}$;

(b) the point $\bar{x}$ is a critical point for $P(t)$, but condition ND1 or ND2 fails to hold [cf. (5), (6)].

In this paper we restrict ourselves basically to problems with equality constraints only, i.e., $J=\varnothing$. In particular, we study some aspects of the set of g.c. points for $P(t)$ as $t$ varies. Although the assumption $J=\varnothing$ seems to be very restrictive, the results in case $J=\varnothing$ form a basic tool for the investigation of the general case $J \neq \varnothing$. We will return to this point in the last section. 


\section{Statement of the Theorems}

In this section, we deal with optimization problems with equality constraints only, and the notations will be appropriately adapted.

Let $n, m$ be fixed integers with $n \geq 1$ and $0 \leq m<n$. Let $f_{0}, f_{1}, \ldots, f_{m}$ be elements of $C^{3}\left(\mathbb{R}^{n+1}, \mathbb{R}\right),(x, t) \mapsto f_{i}(x, t), x \in \mathbb{R}^{n}, t \in \mathbb{R}$. We put

$$
M=\left\{(x, t) \in \mathbb{R}^{n+1} \mid f_{i}(x, t)=0, i=1, \ldots, m\right\}
$$

and

$$
M(t)=\left\{x \in \mathbb{R}^{n} \mid(x, t) \in M\right\} .
$$

For each fixed $t, f_{0}(\cdot, t)$ stands for the objective function, whereas $M(t)$ is the feasible region, determined by the equality constraints $f_{i}(\cdot, t), i=$ $1, \ldots, m$. Together, they define the optimization problem $P(t)$ according to (9). In the following, we use partial derivatives up to third order. Therefore, we assumed all $f_{i}$ to be of the class $C^{3}$. We exclude the case that $M(t)$ is a discrete set for all $t$. This is the reason for taking $m$ less than $n$.

As an abbreviation, we put

$$
\begin{aligned}
& F=\left(f_{0}, f_{1}, \ldots, f_{m}\right)^{\top}, \\
& \operatorname{Coker}(x, t)=\left\{\lambda \in \mathbb{R}^{m+1} \mid \lambda^{\top} D_{x} F(x, t)=0\right\}, \\
& \operatorname{Ker}(x, t)=\left\{\xi \in \mathbb{R}^{n} \mid D_{x} f_{i}(x, t) \xi=0, i=1, \ldots, m\right\} .
\end{aligned}
$$

Here, $D_{x}$ stands for partial derivative with respect to $x$.

Definition 2.1. A point $(x, t) \in \mathbb{R}^{n+1}$ is called a generalized critical point (g.c. point) for $F$ if $(x, t) \in M$ and rank $D_{x} F(x, t) \leq m$. By $\Sigma(F)$, we denote the set of all g.c. points for $F$.

Note that $(x, t) \in \mathbb{R}^{n+1}$ is a g.c. point for $F$ if and only if $x$ is a g.c. point for $f_{0}(\cdot, t)_{\mid M(t)}$ in the sense of Definition 1.1. Given $\lambda \in \mathbb{R}^{m+1}$,

$$
\lambda=\left(\lambda_{0}, \lambda_{1}, \ldots, \lambda_{m}\right)^{\top}
$$

we adjoin to $F$ the following generalized Lagrange function

$$
\mathscr{L}(x, t, \lambda)=\lambda^{\top} F(x, t) .
$$

Let $(\bar{x}, \bar{t})$ be a g.c. point for $F$, with

$$
\operatorname{dim} \operatorname{Coker}(\bar{x}, \bar{t})=1 \text {. }
$$

Let

$$
\bar{\lambda}=\left(\bar{\lambda}_{0}, \bar{\lambda}_{1}, \ldots, \bar{\lambda}_{m}\right)^{\top}
$$

be a generator of $\operatorname{Coker}(\bar{x}, \bar{t})$ and consider the following three special types. 
Type 1. $\quad \bar{\lambda}_{0} \neq 0$; rank $A=n-m$; characteristic number: $\alpha$.

Specification of $A, \alpha$ : Let $V$ be an $n \times(n-m)$-matrix whose columns form a basis for $\operatorname{Ker}(\bar{x}, \bar{t})$ and put

$$
A=\lambda_{0} V^{\top} D_{x}^{2} \mathscr{L} V_{(x, t, \hat{\lambda})=(\bar{x}, \bar{i}, \bar{\lambda})},
$$

where $D_{x}^{2} \mathscr{L}$ stands for the Hessian of $\mathscr{L}$ with respect to $x$. Define $\alpha$ to be the number of negative eigenvalues of $A$.

Type 2. $\bar{\lambda}_{0} \neq 0 ;$ rank $A=n-m-1 ; \beta \neq 0$; characteristic numbers: $\alpha$, $\operatorname{sign}(\beta)$.

Specification of $A, \alpha, \beta$ : The definition of $A$ and $\alpha$ is the same as in Type 1 . Choose $w \in \mathbb{R}^{n-m}, w \neq 0$, such that $A w=0$, and put $v=V w$, with $V$ as in Type 1. Define

$$
F_{0}=\left(f_{1}, \ldots, f_{m}\right)^{\top} \text {. }
$$

Put

$$
\beta_{1}=v^{\top}\left(D_{x}^{3} \mathscr{L} v\right) v-3 v^{\top} D_{x}^{2} \mathscr{L} D_{x}^{\top} F_{0}\left(D_{x} F_{0} D_{x}^{\top} F_{0}\right)^{-1}\left(v^{\top} D_{x}^{2} F_{0} v\right),
$$

where

$$
\begin{aligned}
& v^{\top}\left(D_{x}^{3} \mathscr{L} v\right) v=\sum_{i, j, k=1}^{n}\left(\partial^{3} / \partial x_{i} \partial x_{j} \partial x_{k} \mathscr{L}\right) v_{i} v_{j} v_{k}, \\
& v^{\top} D_{x}^{2} F_{0} v=\left(v^{\top} D_{x}^{2} f_{1} v, \ldots, v^{\top} D_{x}^{2} f_{m} v\right)^{\top},
\end{aligned}
$$

$D_{x} F_{0}$ being the $m \times n$ matrix with $\partial / \partial x_{i} F_{0}$ as $i$ th column, and where all partial derivatives are evaluated at $(\bar{x}, \bar{t}, \bar{\lambda})[$ resp., $(\bar{x}, \bar{t})]$. Put

$$
\beta_{2}=D_{t}\left(D_{x} \mathscr{L} v\right)-\left(D_{t}^{\top} F_{0}\right)\left(D_{x} F_{0} D_{x}^{\top} F_{0}\right)^{-1} D_{x} F_{0} D_{x}^{2} \mathscr{L} v,
$$

where $D_{\mathrm{t}}=\partial / \partial t$ and all partial derivatives are evaluated at $(\bar{x}, \bar{t}, \bar{\lambda})$ [resp., $(\bar{x}, \bar{t})]$. Finally, put

$$
\beta=\beta_{1} \beta_{2} \text {. }
$$

Type 3. $\bar{\lambda}=0 ; B$ nonsingular; $\delta \neq 0$; characteristic numbers: $\gamma$, $\operatorname{sign}(\delta)$. Specification of $B, \gamma, \delta$ : Let $W$ be an $n \times(n-m+1)$ matrix whose columns form a basis for $\operatorname{Ker}(\bar{x}, \bar{t})$, and put

$$
B=D_{t} \mathscr{L} W^{\top} D_{x}^{2} \mathscr{L} W,
$$

evaluated at $(\bar{x}, \bar{t}, \bar{\lambda})$. The number $\gamma$ is defined to be the number of positive eigenvalues of $B$ and

$$
\delta=w^{\top} B^{-1} w
$$

where $w=W^{\top} D_{x}^{\top} f_{0}$, evaluated at $(\bar{x}, \bar{t})$. 
Note that the numbers $\alpha, \operatorname{sign}(\beta), \gamma, \operatorname{sign}(\delta)$ in the above types are independent from the incidental choice of the generator $\vec{\lambda}$ of $\operatorname{Coker}(\bar{x}, \bar{t})$, of $V$, etc. Therefore, we call them characteristic numbers. In the following definition, we identify $C^{3}\left(\mathbb{R}^{n+1}, \mathbb{R}^{m+1}\right)$ with $C^{3}\left(\mathbb{R}^{n+1}, \mathbb{R}\right)^{m+1}$.

Definition 2.2. An $F \in C^{3}\left(\mathbb{R}^{n+1}, \mathbb{R}^{m+1}\right)$ is said to be regular if the following two properties hold for every $(x, t) \in \Sigma(F)$ :

(i) $\operatorname{dim} \operatorname{Coker}(x, t)=1$;

(ii) $(x, t)$ is either of Type 1, Type 2 , or Type 3.

The set $\mathscr{F}$ is defined to be the set consisting of all regular $F$.

Theorem 2.1. (Genericity). $\mathscr{F}$ is $C_{S^{-}}^{3}$ open and dense in $C^{3}\left(\mathbb{R}^{n+1}, \mathbb{R}^{m+1}\right)$.

Theorem 2.2. (Manifold). Let $F \in \mathscr{F}$, and let $\Sigma(F)$ be nonempty. Then, $\Sigma(F)$ is a one-dimensional $C^{2}$-submanifold of $\mathbb{R}^{n+1}$. All critical points of $\phi_{\Sigma(F)}$ are nondegenerate, where $\phi(x, t):=t$. Moreover, a point $(x, t) \in \Sigma(F)$ is a critical point for $\phi_{\mid \Sigma(F)}$ if and only if $(x, t)$ is of Type 2 or Type 3.

Let $F$ be an element of $\mathscr{F}$. If $(x, t) \in \Sigma(F)$ is of Type 1 , then, locally around $x, M(t)$ is a $C^{3}$-manifold and $x$ is a nondegenerate critical point for $f_{0}(\cdot, t)_{\mid M(t)}$. Moreover, the quadratic index (Morse index) at $x$ for $f_{0}(\cdot, t)_{\mid M(t)}$ equals the characteristic number $\alpha$. If $(x, t) \in \Sigma(F)$ is of Type 2 or Type 3 , it is easily seen from Theorem 2.2 that $\Sigma(F)$ can be locally

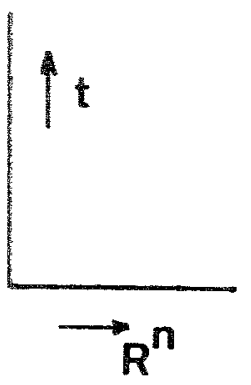

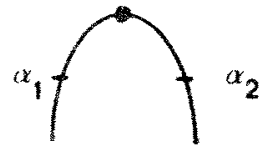

$$
\begin{aligned}
& \alpha_{1}=\alpha \\
& a_{2}=\alpha+1
\end{aligned}
$$

type $2, \operatorname{sign} \beta=+1$

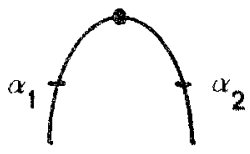

$$
\alpha_{1}=\gamma-1
$$$$
x_{2}=n-m-r+1
$$

type $3, \operatorname{sign} \delta=+1$

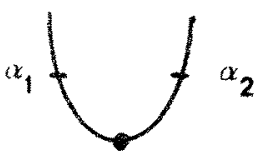

$a_{1}=\alpha$

$\alpha_{2}=\alpha+1$

type $2, \operatorname{sign} \beta=-1$

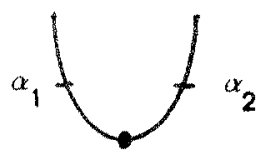

$\alpha_{1}=\gamma$

$\alpha_{2}=n-m-\gamma$

type $3, \operatorname{sign} \delta=-1$

Fig. 1. 
approximated by a parabola. In fact, this parabola is the second-order approximation of the orthogonal projection of $\Sigma(F)$ to the osculating plane of $\Sigma(F)$ at $(x, t)$.

Theorem 2.3. (Quadratic Index). Let $F \in \mathscr{F}$, and denote by $\Sigma_{1}(F)$ the subset of $\Sigma(F)$ consisting of all points of Type 1 . Then, the quadratic index is constant on each connected component of $\Sigma_{1}(F)$. Moreover, passing on $\Sigma(F)$ a point of Type 2 or Type 3 , the quadratic index changes as indicated in Fig. 1.

\section{Proof of the Theorems}

We adopt the notations of Section 2. In particular, $\phi: \mathbb{R}^{n+1} \rightarrow \mathbb{R}$ will always stand for the projection $(x, t) \mapsto t$. We firstly clarify the theorems in two special cases. These two special cases will provide, together with an analysis in local coordinates, the tools for proving the theorems.

Case 1. $\boldsymbol{m}=0$. In this case, we have $F=f_{0}$ and $\Sigma(F)$ reduces to the set $\left\{(x, t) \in \mathbb{R}^{n+1} \mid D_{x} F(x, t)=0\right\}$. By $\mathscr{A}_{i}$ we denote the set of all real symmetric $n \times n$ matrices of rank $i$. Then, $\mathscr{A}_{i}$ is a smooth submanifold of $\mathbb{R}^{\frac{1}{2}(n+1)}$ of codimension $\frac{1}{2}(n-i)(n-i+1)$ (see Ref. 10). Consider the manifolds

$$
\mathscr{M}_{i}=\mathbb{R}^{n} \times \mathbb{R} \times\{0\} \times \mathscr{A}_{i}, \quad i=0,1, \ldots, n,
$$

where $\{0\} \subset \mathbb{R}^{n}$ and the manifold

$$
\mathscr{M}_{F}=\left\{\left(x, t, D_{x} F, D_{x}^{2} F\right) \mid(x, t) \in \mathbb{R}^{n+1}\right\} .
$$

Here, $D_{x}^{2} F$ stands for the matrix $\left(\partial^{2} / \partial x_{i} \partial x_{j} F(x, t)\right)$, viewed at as an element of $\mathbb{R}^{\frac{1}{2} n(n+1)}$. Put

$$
p=2 n+\frac{1}{2} n(n+1)+1 .
$$

Then, $\mathcal{M}_{i}, \mathcal{M}_{F}$ are submanifolds of $\mathbb{R}^{p}$. We say that $F$ satisfies Condition (*) if

$$
\mathscr{M}_{F} \pi \mathscr{M}_{i}, i=0,1, \ldots, n,
$$

where $\pi$ stands for transversal intersection (cf. Ref. 8). Suppose that $F$ satisfies Condition ( $*$ ). Then, in particular, $0 \in \mathbb{R}^{n}$ is a regular value (Ref. 8) for the $C^{2}$-map $(x, t) \mapsto D_{x} F(x, t)$. Consequently, the closed set $\Sigma(F)$ is a one-dimensional $C^{2}$-submanifold of $\mathbb{R}^{n+1}$. Furthermore,

$$
\mathscr{M}_{F} \cap \mathcal{M}_{i}=\varnothing, \quad \text { for } i \leq n-2 .
$$

In fact, note that the inequality $i \leq n-2$ implies that

$$
\operatorname{codim} \mathcal{M}_{i} \geq n+3
$$


(codimension in $\mathbb{R}^{p}$ ). Consequently, in case $D_{x}^{2} F(\bar{x}, \bar{t})$ is singular at $(\bar{x}, \bar{t}) \in$ $\Sigma(F)$, exactly one eigenvalue of $D_{x}^{2} F(\bar{x}, \bar{t})$ vanishes. Now, suppose that $(\bar{x}, \bar{t}) \in \Sigma(F)$ and $\operatorname{det}\left(D_{x}^{2} F(\bar{x}, \bar{t})\right)=0$. From the fact that $\mathscr{M}_{F} \pi \mathscr{M}_{n-1}$, it is not difficult to see that $\operatorname{det}\left(D_{x}^{2} F\right)$ changes sign if we pass $(\bar{x}, \bar{t})$ along $\Sigma(F)$. Moreover, a short but tedious calculation shows that $(\bar{x}, \bar{t})$ is a nondegenerate critical point for $\phi_{\mid \Sigma(F)}$ and that the second derivative of $\phi_{\mid \Sigma(F)}$ in local coordinates of $\Sigma(F)$ at $(\bar{x}, \bar{t})$ is equal to

$$
-\mu v^{\top}\left(D_{x}^{3} F v\right) v D_{t}\left(D_{x} F v\right)
$$

where $v$ is a unit eigenvector belonging to the vanishing eigenvalue of $D_{x}^{2} F$ and $\mu$ some positive scalar. We put

$$
\beta_{1}=v^{\top}\left(D_{x}^{3} F v\right) v, \quad \beta_{2}=D_{i}\left(D_{x} F v\right), \quad \beta=\beta_{1} \beta_{2} .
$$

In particular, if $\beta>0$ [resp., $\beta<0$ ], then $(\bar{x}, \bar{t})$ is a local maximum [resp., local minimum] for $\phi_{\Sigma(F)}$. Note that this is in accordance with Fig. 1. Finally, we put

$$
\mathscr{F}=\left\{F \in C^{3}\left(\mathbb{R}^{n+1}, \mathbb{R}\right) \mid F \text { satisfies Condition (*) }\right\} .
$$

From the above discussion the validity of Theorems 2.2 and 2.3 for the family $\mathscr{F}$ follows at once. Next, we establish that $\mathscr{F}$ is $C_{s}^{3}$-open and dense in $C^{3}\left(\mathbb{R}^{n+1}, \mathbb{R}\right)$, thereby showing that Theorem 2.1 is valid as well. The open part is easily shown with a continuity argument, taking into account that the set $\bigcup_{i=0}^{n-2} \mathscr{A}_{i}$ is closed. Since $C^{\infty}\left(\mathbb{R}^{n+1}, \mathbb{R}\right)$ is $C_{S}^{3}$-dense in $C^{3}\left(\mathbb{R}^{n+1}, \mathbb{R}\right)$ (see Ref. 8), for the dense part it suffices to show that $\mathscr{F} \cap C^{\infty}\left(\mathbb{R}^{n+1}, \mathbb{R}\right)$ is $C_{S}^{3}$-dense in $C^{\infty}\left(\mathbb{R}^{n+1}, \mathbb{R}\right)$. So, let $F \in C^{\infty}\left(\mathbb{R}^{n+1}, \mathbb{R}\right)$. By means of local perturbations of $F$ with polynomials up to degree two, we may achieve [by application of Sard's theorem (Ref. 8) with respect to the coefficients of the perturbation polynomials] that $F$ satisfies Condition $(*)$ locally. The globalization of this local result is carried out by means of a local $\rightarrow$ global construction, as is usually done in differential topology.

Case 2. $m=1$ and at $(\bar{x}, \bar{t})$ the following assumptions hold:

(a) $f_{1}=0, D_{x} f_{1}=0, D_{t} f_{1} D_{x}^{2} f_{1}$ nonsingular;

(b) $D_{x} f_{0}\left(D, f_{1} D_{x}^{2} f_{1}\right)^{-1} D_{x}^{\top} f_{0} \neq 0$.

In this case, we investigate the set $\Sigma(F)$ in a neighborhood of $(\bar{x}, \bar{t})$. Assumption (b) implies, in particular, that $D_{x} f_{0}(\bar{x}, \bar{t}) \neq 0$. Now, a moment of reflection shows that, locally around $(\bar{x}, \bar{t})$, the set $\Sigma(F)$ can be described as the projection onto the first $n+1$ coordinates of the solution set of the following system of $n+1$ equations in the $n+2$ variables $(x, t, \lambda)$ :

$$
\begin{aligned}
\lambda D_{x}^{\top} f_{0}+D_{x}^{\top} f_{1} & =0, \\
f_{1} & =0 .
\end{aligned}
$$


The Jacobian matrix of the system (13), with respect to $(x, t, \lambda)$, at $(\bar{x}, \bar{t}, 0)$ has the following form:

$$
\left[\begin{array}{c:c:c}
D_{x}^{2} f_{1} & D_{t} D_{x}^{\top} f_{1} & D_{x}^{\top} f_{0} \\
\hdashline 0 & D_{t} f_{1} & 0
\end{array}\right]
$$

From Assumption (a) and the fact that $D_{x} f_{0} \neq 0$, it follows from (14) that the solution set of (13), say $\mathcal{M}$, can be parametrized (implicit function theorem) by $\lambda$, but also by some of the $x$-coordinates. In particular, $M$ is, locally around $(\bar{x}, \bar{t}, 0)$, a one-dimensional submanifold of $\mathbb{R}^{n+2}$ of class $C^{2}$. The tangent space $\mathscr{T}$ of $\mathscr{M}$ at $(\bar{x}, \bar{t}, 0)$ is generated by the vector $\left(\xi^{\top}, \eta, \nu\right)^{\top}$, where

$$
\left(\xi^{\top}, \eta, \nu\right)=\left(-D_{x} f_{0} D_{x}^{2} f_{1}^{-1}, 0,1\right) .
$$

To see this, note that $\left(\xi^{\top}, \eta, \nu\right)$ is a solution of the following linear system [cf. (14)]:

$$
\begin{aligned}
D_{x}^{2} f_{1} \xi+\eta D_{t} D_{x}^{\top} f_{1}+\nu D_{x}^{\top} f_{0} & =0 \\
\eta D_{t} f_{1} & =0 .
\end{aligned}
$$

Since $D_{t} f_{1} \neq 0$, we obtain $\eta=0$, and hence

$$
\xi=-\nu D^{2} f_{1}^{-1} D_{x}^{\top} f_{0} \text {. }
$$

This implies (15). Consider the special height function $\Phi(x, t, \lambda)=t$. Then, $(\bar{x}, \bar{t}, 0)$ is a critical point for $\Phi_{\mid \mathcal{A}}$, and the corresponding Lagrange parameters $\alpha_{1}, \ldots, \alpha_{n}, \alpha_{n+1}$ satisfy the following system of linear equations:

$$
\left[\begin{array}{c}
0 \\
\vdots \\
0 \\
1 \\
0
\end{array}\right]=\left[\begin{array}{cc}
D_{x}^{2} f_{1} & 0 \\
D_{t} D_{x} f_{1} & D_{1} f_{1} \\
D_{x} f_{0} & 0
\end{array}\right]\left[\begin{array}{c}
\alpha_{1} \\
\vdots \\
\alpha_{n+1}
\end{array}\right] .
$$

From (17), we see that

$$
\alpha_{1}=\cdots=\alpha_{n}=0,
$$

since $D_{x}^{2} f_{1}$ is nonsingular. Consequently,

$$
\alpha_{n+1}=\left(D_{2} f_{1}\right)^{-1} \text {. }
$$

The associated Lagrange function becomes $t-\alpha_{n+1} f_{1}(x, t)$. The restriction of the Hessian of this Lagrange function to the tangent space $\mathscr{T}$ of $\mathcal{M}$ at $(\bar{x}, \vec{t}, 0)$ becomes (noting that the Lagrange function does not depend on $\lambda)$ :

$$
\begin{gathered}
\left(-D_{x} f_{0} D_{x}^{2} f_{1}^{-1}\right)\left(-\alpha_{n+1} D_{x}^{2} f_{1}\right)\left(-D_{x} f_{0} D_{x}^{2} f_{1}^{-1}\right)^{\top} \\
=-D_{x} f_{0}\left(D_{t} f_{1} D_{x}^{2} f_{1}\right)^{-1} D_{x}^{\top} f_{0}=:-\delta .
\end{gathered}
$$


Note that $\delta$ in (18) does not vanish in view of Assumption (b). In particular, if $\delta>0$ [resp., $\delta<0$ ], then $(\bar{x}, \bar{t}, 0)$ is a local maximum [resp., local minimum] for $\Phi_{\mid k}$. From the fact that $\mathscr{M}$ can be parametrized by some $x$-coordinate, it is easily seen that $\Sigma(F)$ is, locally around $(\bar{x}, \bar{t})$, a $C^{2}$-submanifold of $\mathbb{R}^{n+1}$ and that $(\bar{x}, \bar{t})$ is a nondegenerate critical point for $\phi_{\mid \Sigma(F)}$. Moreover, the second derivative of $\phi_{\mid \Sigma(F)}$ in local coordinates of $\Sigma(F)$ is a positive multiple of $(-\delta)$ in (18). Compare this with the orientation of the parabolas (Type 3) in Fig. 1.

We proceed with a discussion of the case $\delta>0$. Then, for $\tilde{t}, \tilde{t}<\bar{t}$ and $\tilde{t} \approx \bar{t}$, there are exactly two solutions $(x, \tilde{t}, \lambda)$ of $(13)$ in a neighborhood of $(\bar{x}, \bar{t}, 0)$, say $\left(\tilde{x}^{1}, \tilde{t}, \tilde{\lambda}^{1}\right),\left(\tilde{x}^{2}, \tilde{t}, \tilde{\lambda}^{2}\right)$. Since a generator for the tangent space $\mathscr{T}$ of $\mathscr{M}$ at $(\bar{x}, \bar{t}, 0)$ has some nonvanishing $x$-component and a nonvanishing $\lambda$-component [cf. (15)], we see that $\tilde{x}^{1} \neq \tilde{x}^{2}($ and $\neq \bar{x})$ and that $\tilde{\lambda}^{1}, \tilde{\lambda}^{2}$ are both nonvanishing and of opposite sign. The points $\tilde{x}^{i}, i=1,2$, are critical points for $f_{0}(\cdot, \tilde{t})$ restricted to the set $\left\{x \mid f_{1}(x, \tilde{t})=0\right\}$ with $-1 / \tilde{\lambda}^{i}$ as corresponding Lagrange parameter [cf. (13)]. In order to compute the corresponding Morse index at $\tilde{x}^{i}$, we have to determine the number of negative eigenvalues of the following restricted matrix at $\left(\tilde{x}^{i}, \tilde{t}\right)$ :

$$
D_{x}^{2}\left[f_{0}+1 / \tilde{\lambda}^{i} D f_{1}\right]_{\mid \operatorname{Ker} D_{x} f_{1}},
$$

where

$$
\operatorname{Ker} D_{x} f_{1}=\left\{\xi \in \mathbb{R}^{n} \mid D_{x} f_{1}\left(\tilde{x}^{i}, \tilde{t}\right) \xi=0\right\} .
$$

Since $\left|\tilde{\lambda}^{i}\right|,\left\|\tilde{x}^{i}-\bar{x}\right\|,|\tilde{t}-\tilde{t}|$ are arbitrarily small, and since

$\operatorname{Ker} D_{x} f_{1}=\operatorname{Ker} D_{x} f_{0}$

in (19), we may equally well determine the number of negative eigenvalues of the following restricted matrices:

$$
\pm D_{x}^{2} f_{1}(\bar{x}, \bar{t})_{\mid \operatorname{Ker} D_{x} f_{0}(\bar{x}, \bar{i})},
$$

or equivalently of the following matrices:

$$
\pm D_{i} f_{1}(\bar{x}, \bar{t}) D_{x}^{2} f_{1}(\bar{x}, \bar{t})_{\mid \operatorname{Kex} D_{x} f_{0}(\hat{x}, \bar{i})},
$$

provided that the restricted matrices in (20) [resp., (21)] are nonsingular. This latter fact, however, follows from the Assumptions (a), (b) and the following lemma.

Lemma 3.1. Let $B$ be a real nonsingular symmetric $n \times n$ matrix, $w \in \mathbb{R}^{n}$, and define

$$
w^{\perp}=\left\{\xi \in \mathbb{R}^{n} \mid w^{\top} \xi=0\right\} .
$$

Let the word index stand for the number of negative eigenvalues. Then:

(a) if $w^{\top} B^{-1} w \neq 0, B_{\mid w^{\perp}}$ is nonsingular; 
(b) if $w^{\top} B^{-1} w>0$, index $B_{\mid w^{\perp}}=\operatorname{index} B$; if $w^{\top} B^{-1} w<0$, index $B_{\mid w^{\perp}}=$ index $B-1$.

Proof. Let

$w^{\top} B^{-1} w \neq 0$,

and let $V$ be an $n \times(n-1)$ matrix whose columns span $w^{\perp}$. Put

$$
W=[B V: w] \text {. }
$$

Then, we have

$$
W^{\top} B^{-1} W=\left[\begin{array}{c:c}
V^{\top} B V & 0 \\
\hdashline 0 & w^{\top} B^{-1} w
\end{array}\right] .
$$

The matrix $V^{\top} B V$ is nonsingular. In fact, suppose that

$$
V^{\top} B V \alpha=0, \text { for some } \alpha \in \mathbb{R}^{n-1} \text {. }
$$

Then,

$$
B V \alpha=\gamma w, \quad \text { for some } \gamma \in \mathbb{R} .
$$

Hence,

$$
V \alpha=\gamma B^{-1} w,
$$

and thus

$$
0=w^{\top} V \alpha=\gamma w^{\top} B^{-1} w .
$$

This implies that $\gamma=0$. But then, $V \alpha=0$, and consequently $\alpha=0$. This implies the nonsingularity of $V^{\top} B V$, and hence Statement (a) of Lemma 3.1. From the block structure in (22), we now conclude that $W$ is nonsingular. Hence, in view of Sylvester's theorem (Ref. 4), we have

$$
\text { index } W^{\top} B^{-1} W=\text { index } B^{-1} \text {. }
$$

Obviously,

$$
\text { index } B^{-1}=\text { index } B
$$

and

index $V^{\top} B V=$ index $B_{\mid w^{+}}$.

Now, Statement (b) of Lemma 3.1 follows by taking the block structure (22) into account.

Now, we put

$$
B=D_{t} f_{1}(\bar{x}, \bar{t}) D_{x}^{2} f_{1}(\bar{x}, \bar{t}), \quad w=D_{x}^{\top} f_{0}(\bar{x}, \bar{t}),
$$


and let $\gamma$ be the number of positive eigenvalues of $B$. Thus, index $B=n-\gamma$.

Since we still assume $\delta>0$, we have

$$
w^{\top} B^{-1} w>0
$$

[cf. (18)]. From Lemma 3.1, it follows that

$$
\text { index } B_{\mid w^{\perp}}=\text { index } B=n-\gamma \text {. }
$$

For the matrix $(-B)$ we have

$$
\text { index }(-B)=\gamma
$$

and

$$
w^{\top}(-B)^{-1} w<0 \text {. }
$$

Again from Lemma 3.1 it follows that

$$
\text { index }\left(-B_{\mid w^{+}}\right)=\text {index }(-B)-1=\gamma-1 \text {. }
$$

From this discussion, it follows that the Morse index at $\tilde{x}^{i}$ equals $n-\gamma$ [resp., $\gamma-1$ ]. This is in accordance with Fig. 1 [Type $3, \operatorname{sign}(\delta)=+1, m=1$ ]. The calculation for the case $\delta<0$ is analogous and will be omitted.

In order to exploit the foregoing two special cases within a local coordinate analysis, we need two more lemmas.

Put

$$
M\left[f_{1}, \ldots, f_{m}\right]=\left\{(x, t) \in \mathbb{R}^{n+1} \mid f_{i}(x, t)=0, i=1, \ldots, m\right\} .
$$

If the functions $f_{1}, \ldots, f_{m}$ are fixed, then we write $M$ instead of $M\left[f_{1}, \ldots, f_{m}\right]$. The subset $\mathscr{G}^{*}$ of $C^{3}\left(\mathbb{R}^{n+1}, \mathbb{R}^{m}\right)$ is defined as follows:

$$
\begin{aligned}
\mathscr{G}^{*}= & \left\{\left(f_{1}, \ldots, f_{m}\right)^{\top} \in C^{3}\left(\mathbb{R}^{n+1}, \mathbb{R}^{m}\right) \mid\right. \\
& \left.\phi_{\mid M\left[f_{1}, \ldots, f_{m}\right]} \text { is regular in the sense of Definition } 1.5\right\} .
\end{aligned}
$$

Lemma 3.2. $\quad \mathscr{G}^{*}$ is $C_{S^{-}}^{3}$ open and dense in $C^{3}\left(\mathbb{R}^{n+1}, \mathbb{R}^{m}\right)$.

In Ref. 11 (Lemma 1.1), it is shown that $\mathscr{G}^{*}$ is $C_{S^{2}}^{2}$ open and dense in $C^{\infty}\left(\mathbb{R}^{n+1}, \mathbb{R}^{m}\right)$. However, the same arguments can be used in order to establish Lemma 3.2, taking into account that $C^{\infty}\left(\mathbb{R}^{n+1}, \mathbb{R}^{m}\right)$ is $C_{S}^{3}$-dense in $C^{3}\left(\mathbb{R}^{n+1}, \mathbb{R}^{m}\right)$.

Next, we put

$$
\mathscr{F}^{*}=C^{3}\left(\mathbb{R}^{n+1}, \mathbb{R}\right) \times \mathscr{G}^{*} .
$$

Then, $\mathscr{F}^{*}$ is $C_{S}^{3}$-open and dense in $C^{3}\left(\mathbb{R}^{n+1}, \mathbb{R}^{m+1}\right)$. The set $\mathscr{F}$ in Definition 2.2 will actually become a subset of $\mathscr{F}^{*}$. The proof of the next lemma is easy and will be omitted. 
Lemma 3.3. Let $F \in \mathscr{F}^{*}$ and $(\bar{x}, \bar{t}) \in M$. Then:

(a) if $(\bar{x}, \bar{t})$ is not a critical point for $\phi_{\mid M},\left\{D_{x} f_{i}, i=1, \ldots, m\right\}$ is a linearly independent set;

(b) if $(\bar{x}, \bar{t})$ is a critical point for $\phi_{\mid M}$,

$\operatorname{dim} \operatorname{span}\left\{D_{x} f_{i}, i=1, \ldots, m\right\}=m-1 ;$

furthermore, there exist unique reals $\alpha_{i}, i=1, \ldots, m$, such that

$$
\begin{aligned}
& \sum_{i=1}^{m} \alpha_{i} D_{x} f_{i}=0, \quad \sum_{i=1}^{m} \alpha_{i} D_{t} f_{i}=1, \\
& D_{x}^{2}\left(\sum_{i=1}^{m} \alpha_{i} f_{i}\right)_{\mid \operatorname{Ker}(\bar{x}, \bar{t})} \text { nonsingular. }
\end{aligned}
$$

Let $F \in \mathscr{F}^{*}$, and let $(\bar{x}, \bar{t}) \in M$ be a critical point for $\phi_{\mid M}$. Then, $(\bar{x}, \bar{t})$ belongs to $\Sigma(F)$, and we may assume without loss of generality that $\left\{D_{x} f_{1}, \ldots, D_{x} f_{m-1}\right\}$ is a linearly independent set [cf. Lemma 3.3(b)]. This implies that $\alpha_{m}$ in (23) does not vanish. Choose $\xi_{m}, \xi_{m+1}, \ldots, \xi_{n} \in \mathbb{R}^{n}$ such that $\left\{D_{x}^{\top} f_{1}, \ldots, D_{x}^{\top} f_{m-1}, \xi_{m}, \ldots, \xi_{n}\right\}$ forms a basis for $\mathbb{R}^{n}$, and define the $C^{3}$-map $Q$ as follows:

$$
Q(x, t)=\left(f_{1}(x, t), \ldots, f_{m-1}(x, t), \xi_{m}^{\top}(x-\bar{x}), \ldots, \xi_{n}^{\top}(x-\bar{x}), t-\bar{t}\right)^{\top} .
$$

Then,

$$
Q(\bar{x}, \bar{t})=0
$$

and $D Q(\bar{x}, \bar{t})$ nonsingular. Hence, $Q$ is a local coordinate transformation. By $(y, u)$ we denote the new coordinates. Note that $Q$ maps $t$-hyperplanes to $u$-hyperplanes and that $Q$ preserves the orientation of the $t$-axis. In the $(y, u)$-coordinates, the set $M$ is locally represented by the following set:

$$
\left\{(y, u) \mid \alpha_{m} f_{m} \circ Q^{-1}(y, u)=0, y_{1}=\cdots=y_{m-1}=0\right\} \text {. }
$$

A short calculation shows that

$$
\begin{aligned}
& \left(\partial / \partial y_{i}\right) \alpha_{m} f_{m} \circ Q^{-1}(0)=0, \quad i=m, m+1, \ldots, n, \\
& {\left[\left(\partial^{2} / \partial y_{i} \partial y_{j}\right) \alpha_{m} f_{m} \circ Q^{-1}(0)\right]_{i, j=m, m+1, \ldots, n}=W^{\top} D_{x}^{2}\left[\sum_{i=1}^{m} \alpha_{i} f_{i}(\bar{x}, \bar{t})\right] W,}
\end{aligned}
$$

where $W$ is a suitably chosen $n \times(n-m+1)$ matrix whose columns span $\operatorname{Ker}(\bar{x}, \bar{t})$,

$$
\begin{aligned}
& (\partial / \partial u) \alpha_{m} f_{m} \circ Q^{-1}(0)=D_{t}\left[\sum_{i=1}^{m} \alpha_{i} f_{i}(\bar{x}, \bar{t})\right], \\
& \left(\partial / \partial y_{m}, \ldots, \partial / \partial y_{n}\right) f_{0} \circ Q^{-1}(0)=D_{x} f_{0}(\bar{x}, \bar{t}) W,
\end{aligned}
$$

where $W$ coincides with the matrix $W$ in (27). 
Put

$$
\begin{aligned}
& B=D_{t}\left[\sum_{i=1}^{m} \alpha_{i} f_{i}(\bar{x}, \bar{t})\right] W^{\top} D_{x}^{2}\left[\sum_{i=1}^{m} \alpha_{i} f_{i}(\bar{x}, \bar{t})\right] W, \\
& w=W^{\top} D_{x}^{\top} f_{0}(\bar{x}, \bar{t}) .
\end{aligned}
$$

From Lemma 3.3(b) it follows that $B$ is nonsingular. By means of a local perturbation of $f_{0}$ in a neighborhood of $(\bar{x}, \bar{t})$ with a suitable linear function, we can achieve that $w^{\top} B^{-1} w \neq 0$. Note that such a perturbation can be done simultaneously at all critical points of $\phi_{\mid M}$, since the critical points of $\phi_{\mid M}$, being nondegenerate, are isolated.

Concerning the above analysis in local coordinates, we see that we are in the situation of Case 2, with space dimension $n-m+1$ instead of $n$. Moreover, the sketched perturbation argument implies the following lemma.

Lemma 3.4. Define $\mathscr{F}^{* *}$ as follows:

$$
\mathscr{F}^{* * *}=\left\{F \in \mathscr{F}^{*} \mid \text { every critical point }(x, t) \text { for } \phi_{\mid M\left[f_{1}, \ldots, f_{m}\right]}\right. \text { satisfies }
$$

$$
\operatorname{Coker}(x, t)=1 \text { and }(x, t) \text { is of Type } 3 \text { for } \Sigma(F)\} \text {. }
$$

Then, $\mathscr{F}^{* *}$ is $C_{S}^{3}$-open and dense in $C^{3}\left(\mathbb{R}^{n+1}, \mathbb{R}^{m+1}\right)$.

Now, suppose that $F \in \mathscr{F}^{* * *}$ and that $(\bar{x}, \bar{t}) \in M$ is not a critical point for $\phi_{\mid M}$. Since $\mathscr{F}^{* *} \subset \mathscr{F}^{*}$, it follows from Lemma 3.3(a) that $\left\{D_{x} f_{i}, i=\right.$ $1, \ldots, m\}$ is a linearly independent set. Choose vectors $\xi_{m+1}, \ldots, \xi_{n} \in \mathbb{R}^{n}$ such that $\left\{D_{x}^{\top} f_{1}, \ldots, D_{x}^{\top} f_{m}, \xi_{m+1}, \ldots, \xi_{n}\right\}$ forms a basis for $\mathbb{R}^{n}$, and define the $C^{3}$-map $Q$ as follows:

$$
Q(x, t)=\left(f_{1}(x, t), \ldots, f_{m}(x, t), \xi_{m+1}^{\top}(x-\bar{x}), \ldots, \xi_{n}^{\top}(x-\bar{x}), t-\bar{t}\right)^{\top} .
$$

Then, $Q(\bar{x}, \bar{t})=0, D Q(\bar{x}, \bar{t})$ nonsingular, and hence $Q$ is a local coordinate transformation. By $(y, u)$ we denote the new coordinates. Note that, again, $Q$ maps $t$-hyperplanes to $u$-hyperplanes and that $Q$ preserves the orientation of the $t$-axis. In the $(y, u)$-coordinates, the set $M$ is locally represented by the equations

$$
y_{1}=\cdots=y_{m}=0 \text {. }
$$

Put $\tilde{y}=\left(y_{m+1}, \ldots, y_{n}\right)$ and

$$
\tilde{f}_{0}(\tilde{y}, u)=f_{0} \circ Q^{-1}(0, \tilde{y}, u) \text {. }
$$

By means of (32) we have reduced our analysis, locally, to Case 1, with space dimension $n-m$ instead of $n$. From the discussion in Case 1, we see that we can approximate $\tilde{f}_{0}$ arbitrarily well, locally, in the $C^{3}$-sense such that the approximating function satisfies, again locally, Condition (*) as 
introduced in Case 1. But then, it is easily seen that we can extend this approximation to a local approximation of $f_{0}$ itself in the original coordinates. This implies immediately the following lemma.

Lemma 3.5. Define $\mathscr{F}$ as follows:

$\mathscr{F}=\left\{F \in \mathscr{F}^{* *} \mid\right.$ at every point $(\bar{x}, \bar{t}) \in M$, with $(\bar{x}, \bar{t})$ not a critical point for $\phi_{\mid M}$, the function $\tilde{f}_{0}$ in (32), according to local coordinates of the form (30), satisfies Condition (*) as introduced in Case 1 \}.

Then, $\mathscr{F}$ is $C_{S}^{3}$-open and dense in $C^{3}\left(\mathbb{R}^{n+1}, \mathbb{R}^{m+1}\right)$.

In fact, the family $\mathscr{F}$ in Lemma 3.5 is precisely the family $\mathscr{F}$ as introduced in Definition 2.2.

As a matter of fact, the remaining part of the proof of the theorems basically consists of the clarification of the formulas for $\beta_{1}, \beta_{2}$ in the specification of Type 2 (Section 2). More specifically, we have to determine the numbers $\beta_{1}, \beta_{2}$ in (12) with $F:=\tilde{f}_{0}$ in terms of the functions $f_{0}, f_{1}, \ldots, f_{m}$.

The assumptions on $\tilde{f}_{0}$ are:

$D_{\tilde{y}} \tilde{f}_{0}(0)=0, \quad \operatorname{rank} D_{\tilde{y}}^{2} \tilde{f}_{0}(0)=n-m-1$.

Let $w$ be a nonvanishing $(n-m)$-vector such that

$$
D_{\tilde{y}}^{2} \tilde{f}_{0}(0) w=0 .
$$

From (33), it follows that there are unique numbers $\alpha_{1}, \ldots, \alpha_{m}$ such that

$$
D_{x} f_{0}+\sum_{i=1}^{m} \alpha_{i} D_{x} f_{i}=0_{\mid(\bar{x}, \bar{l})} .
$$

Put

$$
L(x, t)=f_{0}(x, t)+\sum_{i=1}^{m} \alpha_{i} f_{i}(x, t) .
$$

Firstly, we calculate the following number [cf. (12)]:

$$
w^{\top}\left(D_{\tilde{y}}^{3} \tilde{f}(0) w\right) w .
$$

A moment of reflection shows that the number in (37) equals

$$
d^{3} / d \gamma^{3} L \circ Q^{-1}(0, \gamma w, 0)_{\mid \gamma=0} .
$$

As an abbreviation, we denote by $x(\gamma)$ the vector consisting of the first $n$ components of $Q^{-1}(0, \gamma w, 0)$. So, we have to determine the number $d^{3} / d \gamma^{3} L(x(\gamma), \bar{t})_{\mid \gamma=0}$. Let $\langle\cdot, \cdot\rangle$ denote the standard inner product. Then,

$$
(d / d \gamma) L(x(\gamma), \bar{t})=\left\langle D_{x}^{\top} L, \dot{x}\right\rangle,
$$


where $\dot{x}[$ resp., $\ddot{x}, \ddot{x}]$ stands for the first [resp., second, third] derivative of $x(\gamma)$.

Furthermore,

$$
\left(d^{2} / d \gamma^{2}\right) L(x(\gamma), \bar{t})=\left\langle D_{x}^{2} L \dot{x}, \dot{x}\right\rangle+\left\langle D_{x}^{\top} L, \ddot{x}\right\rangle ;
$$

hence,

$$
\left(d^{3} / d \gamma^{3}\right) L(x(\gamma), \bar{t})_{\mid \gamma=0}=\left\langle\left(D_{x}^{3} L \dot{x}\right) \dot{x}, \dot{x}\right\rangle+3\left\langle D_{x}^{2} L \dot{x}, \ddot{x}\right\rangle+\left\langle D_{x}^{\top} L, \ddot{x}\right\rangle_{\mid \gamma=0} .
$$

Note that $D_{x} L(\bar{x}, \bar{t})$ vanishes [cf. (35), (36)], and hence the last term on the right in (39) vanishes. A simple calculation shows that

$$
D_{\tilde{y}}^{2} \tilde{f}_{0}(0)=V^{\top} D_{x}^{2} L(\bar{x}, \bar{t}) V, \quad \dot{x}(0)=V w,
$$

where $V$ is a suitably chosen $n \times(n-m)$ matrix whose columns span $\operatorname{Ker}(\bar{x}, \bar{t})$. A combination of (34) and (40) shows that

$$
V^{\top} D_{x}^{2} L(\bar{x}, \bar{t}) V w=0
$$

and hence

$$
V^{\top} D_{x}^{2} L(\bar{x}, \bar{t}) \dot{x}(0)=0 .
$$

Consequently, $D_{x}^{2} L(\bar{x}, \bar{t}) \dot{x}(0)$ is orthogonal to $\operatorname{Ker}(\bar{x}, \bar{t})$. We write

$$
\ddot{x}(0)=\xi+D_{x}^{\top} F_{0} \eta
$$

where $\xi \in \operatorname{Ker}(\bar{x}, \bar{t}), \eta$ a unique $m$-vector, and

$$
F_{0}=\left(f_{1}, \ldots, f_{m}\right)^{\top} \text {. }
$$

With this notation, $\left\langle D_{x}^{2} L \dot{x}, \ddot{x}\right\rangle$ in (39) reduces to $\left\langle D_{x}^{2} L \dot{x}, D_{x}^{\top} F_{0} \eta\right\rangle$. So, it remains to determine $\eta$ in terms of the functions $f_{0}, f_{1}, \ldots, f_{m}$ in order to obtain an explicit formula for (37).

From the definition of $x(\gamma)$, we have

$$
f_{i}(x(\gamma), \bar{t}) \equiv 0, \quad i=1, \ldots, m .
$$

Consequently,

$$
\left\langle D_{x}^{\top} f_{i}, \dot{x}\right\rangle \equiv 0,
$$

and hence

$$
\left\langle D_{x}^{2} f_{i} \dot{x}, \dot{x}\right\rangle+\left\langle D_{x}^{\top} f_{i}, \ddot{x}\right\rangle \equiv 0, \quad i=1, \ldots, m .
$$

As an abbreviation, we put

$$
v=V w(=\dot{x}(0)) .
$$

Then, we obtain

$$
D_{x} F_{0} \ddot{x}(0)=-\left(v^{\top} D_{x}^{2} f_{1} v, \ldots, v^{\top} D_{x}^{2} f_{m} v\right)_{\mid(\bar{x}, \bar{t})}^{\top} .
$$


Furthermore,

$$
D_{x} F_{0} \ddot{x}(0)=D_{x} F_{0}\left(\xi+D_{x}^{\top} F_{0} \eta\right)=D_{x} F_{0} D_{x}^{\top} F_{0} \eta ;
$$

and hence, using (42) and the fact that $D_{x} F_{0}$ is of full rank, we obtain

$$
\eta=-\left(D_{x} F_{0} D_{x}^{\top} F_{0}\right)^{-1}\left(v^{\top} D_{x}^{2} f_{1} v, \ldots, v^{\top} D^{2} f_{m} v\right)_{\mid(\bar{x}, \bar{\tau})}^{\top} .
$$

A combination of (39), (40), (41), (43) yields the formula for the number $\beta_{1}$ in the specification of Type 2 in Section 2 .

Finally, we have to determine the following number [cf. (12)]:

$$
\left(\partial^{2} / \partial \gamma \partial u\right) \tilde{f}_{0}(\gamma w, u)_{\mid \gamma=u=0} .
$$

A moment of reflection shows that the number in (44) is equal to

$$
\left(\partial^{2} / \partial u \partial \gamma\right) L \circ Q^{-1}(0, \gamma w, u)_{\mid \gamma=u=0} .
$$

In order to compute (45), we firstly note that

$$
(\partial / \partial \gamma) L \circ Q^{-1}(0, \gamma w, u)=\left\langle D^{\top} L, D Q^{-1}\left[\begin{array}{l}
0 \\
w \\
0
\end{array}\right]\right) .
$$

Consequently, the number in (45) becomes

$$
\left\langle D^{2} L D Q^{-1}\left[\begin{array}{l}
0 \\
0 \\
1
\end{array}\right], D Q^{-1}\left[\begin{array}{l}
0 \\
w \\
0
\end{array}\right]\right\rangle+\left\langle D^{\top} L, \partial / \partial u D Q^{-1}\left[\begin{array}{l}
0 \\
w \\
0
\end{array}\right]\right\rangle_{\mid \gamma=u=0} .
$$

Note that

$$
D L=\left(0,0, D_{t} L\right)_{\mid(\bar{x}, \bar{l})} .
$$

Put

$$
\eta(u)=D Q^{-1}(0,0, u)\left[\begin{array}{l}
0 \\
w \\
0
\end{array}\right] .
$$

Then, it is easily seen that the last component of $\eta(u)$ identically vanishes. Consequently, the derivative of the last component of $\eta(u)$ vanishes as well; and hence, using (48), we see that the right term in (47) vanishes. A short calculation shows that

$$
D Q^{-1}(0)\left[\begin{array}{l}
0 \\
w \\
0
\end{array}\right]=\left[\begin{array}{c}
V w \\
0
\end{array}\right]:=\left[\begin{array}{l}
v \\
0
\end{array}\right]
$$


with $V w$ as in (40). So it remains to calculate

$$
D Q^{-1}(0)\left[\begin{array}{l}
0 \\
0 \\
1
\end{array}\right]=\left(\begin{array}{c}
\zeta \\
\zeta_{n+1}
\end{array}\right) \text {. }
$$

Then, $\zeta, \zeta_{n+1}$ solve the following linear system:

$$
\left[\begin{array}{c}
0 \\
\vdots \\
0 \\
1
\end{array}\right]=\left[\begin{array}{cc}
D_{x} f_{1} & D_{t} f_{1} \\
\vdots & \vdots \\
\xi_{m+1}^{\top} & 0 \\
\vdots & \vdots \\
\xi_{n}^{\top} & 0 \\
0 & 1
\end{array}\right]_{(\bar{x}, \bar{i})} \cdot\left[\begin{array}{c}
\zeta \\
\zeta_{n+1}
\end{array}\right] .
$$

From (49), we see that $\zeta_{n+1}=1$. Hence, the left term in (47) becomes

$$
\left\langle\zeta, D_{x}^{2} L v\right\rangle+D_{i}\left(D_{x} L v\right)
$$

Next, we write

$$
\zeta=\xi+D_{x}^{\top} F_{0} \eta
$$

where $\xi \in \operatorname{Ker}(\bar{x}, \bar{t})$ and $\eta$ a unique $m$-vector. Recall that $D_{x}^{2} L(\bar{x}, \bar{t}) v$ is orthogonal to $\operatorname{Ker}(\bar{x}, \bar{t})$. Consequently,

$$
\left\langle\zeta, D_{x}^{2} L v\right\rangle=\left\langle D_{x}^{\top} F_{0} \eta, D_{x}^{2} L v\right\rangle
$$

Since $\xi \in \operatorname{Ker}(\bar{x}, \bar{t})$, we have

$$
D_{x} F_{0} \zeta=D_{x} F_{0} D_{x}^{\top} F_{0} \eta \text {. }
$$

From (49) we see that

$$
D_{x} F_{0} \zeta=-D_{t} F_{0}
$$

and hence, we finally obtain

$$
\eta=-\left(D_{x} F_{0} D_{x}^{\top} F_{0}\right)^{-1} D_{t} F_{0 \mid(\tilde{x}, \vec{z})} .
$$

A combination of (50)-(52) yields the desired formula for $\beta_{2}$ as in the specification of Type 2 (Section 2) and this, at last, completes the proof of our theorems. 


\section{Final Remarks}

In this section, we adopt the general notation of Section 1. In Section 2 and Section 3 we studied in detail the equality constrained case, i.e., $J=\varnothing$. The general case, $J \neq \varnothing$, can be treated as follows. Put

$$
J_{0}(x, t)=\left\{j \in J \mid g_{j}(x, t)=0\right\} .
$$

In a neighborhood of those feasible points $(x, t)$ at which $\left\{D_{x} h_{i}, D_{x} g_{j}, i \epsilon\right.$ $\left.I, j \in J_{0}(x, t)\right\}$ is a linearly independent set, the basic tools for extending the presented theory are given in Ref. 12. In particutar, Ref. 12 contains a detailed study of the case where a Lagrange parameter, corresponding to an active inequality constraint, vanishes.

In the case where the gradients (with respect to $x$ ) of the active constraints are linearly dependent, it turns out that one has to distinguish the following two situations, namely,

$$
|I|+\left|J_{0}(x, t)\right|=n+1
$$

and

$$
|I|+\left|J_{0}(x, t)\right|<n+1 .
$$

The results in Section 2 can be applied in the latter situation, as a complicated generic activity analysis shows. Such an analysis, however, would blow up the size of this paper. It will be the subject of a forthcoming paper (Ref. 13).

Another approach in parametric optimization is presented in the very interesting papers of Kojima (Ref. 5) and Kojima and Hirabayashi (Refs. 14 and 15). In their analysis, they restricted themselves to the study of Kuhn-Tucker points with the Mangasarian-Fromowitz constraint qualification as the underlying assumption. We believe that their approach and ours together will finally result in a good understanding of the topology of nonlinear parametric problems.

\section{References}

1. MCCORMICK, G. P., Optimality Criteria in Nonlinear Programming, SIAM-AMS Proceedings, Vol. 9, pp. 27-38, 1976.

2. Hettich, R., and Jongen, H. TH., On First-Order and Second-Order Conditions for Local Optima for Optimization Problems in Finite Dimensions, Methods of Operations Research, Vol. 23, pp. 82-97, 1977.

3. Milnor, J., Morse Theory, Annals of Mathematic Studies, Study 51, Princeton University Press, Princeton, New Jersey, 1963.

4. Marcus, M., and MINC, H., A Survey of Matrix Theory and Matrix Inequalities, Allyn and Bacon, Boston, Massachusetts, 1964. 
5. KoJimA, M., Strongly Stable Stationary Solutions in Nonlinear Programs, Analysis and Computation of Fixed Points, Edited by S. M. Robinson, Academic Press, New York, New York, 1980.

6. BRAESS, D., Morse Theorie für Berandete Mannigfaltigkeiten, Mathematische Annalen, Vol. 208, pp. 133-148, 1974.

7. Jongen, H. TH., Jonker, P. and Twilt, F., Nonlinear Optimization in $\mathbb{R}^{n}$, I: Morse Theory, Chebyshev Approximation, Peter Lang Verlag, Frankfurt a.M., Germany 1983.

8. HIRSCH, M. W., Differential Topology, Springer Verlag, Berlin, Germany, 1976.

9. JoNGEN, H. TH., JONKER, P., and TWILT, F., Nonlinear Optimization in $\mathbb{R}^{n}, I I$ : Transversality, Flows, Parametric Aspects (to appear).

10. Lu, Y. C., Singularity Theory and an Introduction to Catastrophe Theory, Universitext, Springer Verlag, Berlin, Germany, 1976.

11. Jongen, H. TH., Jonker, P., and TwILT, F., On One-Parameter Families of Sets Defined by (In)Equality Constraints, Nieuw Archief voor Wiskunde, Vol. 30, pp. 307-322, 1982.

12. JoNGEN, H. TH., JONKER, P., and TwILT, F., On Deformation in Optimization, Methods of Operations Research, Vol. 37, pp. 171-184, 1980.

13. Jongen, H. Th., Jonker, P., and TwLt, F., Critical Sets in Parametric Optimization, Mathematical Programming (to appear).

14. Kojma, M., and Hirabayashi, R., Some Results on the Strong Stability in Nonlinear Programs, Tokyo Institute of Technology, Department of Management Science and Engineering, Technical Report No. 4, 1980.

15. Kojima, M., and Hirabayashi, R., Continuous Deformation of Nonlinear Programs, Mathematical Programming Study, Vol. 21, pp. 150-198, 1984. 